\title{
Archéopages
}

Archéopages Archéologie et société

Hors-série 2 | 2010

Archéologie sans frontières

\section{La fouille des ateliers du secteur 2}

\section{Aurore Louis}

\section{OpenEdition}

Journals

Édition électronique

URL : https://journals.openedition.org/archeopages/814

DOI : 10.4000/archeopages. 814

ISSN : 2269-9872

\section{Éditeur}

INRAP - Institut national de recherches archéologiques préventives

\section{Édition imprimée}

Date de publication : 1 octobre 2010

Pagination : 127-129

ISSN : 1622-8545

\section{Référence électronique}

Aurore Louis, «La fouille des ateliers du secteur 2 », Archéopages [En ligne], Hors-série 2 | 2010, mis en ligne le 01 octobre 2010, consulté le 23 février 2023. URL : http://journals.openedition.org/ archeopages/814 ; DOI : https://doi.org/10.4000/archeopages.814 
La capacité de production des ateliers du Wadi Natrun semble énorme, par rapport à ce qui est attesté jusque-là : en effet, dans un four comme celui du secteur 2 , on produisait une dalle qui devait avoisiner les 15 à $20 t$ de verre brut. Cette avancée technologique se déroulait à la même époque qu'une autre révolution : l'invention du verre soufflé et sa diffusion dans toutes les provinces de l'Empire, qui a fait passer les verres de produits de luxe à objets d'usage quotidien. Une fois la publication achevée dans les Travaux de la Maison de l'Orient, deux voies d'exploration s'ouvriront : prospection à la recherche d'ateliers primaires de verriers dans le Sinaï, dont on sait qu'ils ont fonctionné à partir de la fin du $\mathrm{IV}^{\mathrm{e}}$ siècle et dont le verre brut a alimenté l'ensemble des ateliers de l'Empire romain ; prospection dans les environs d'Alexandrie à la recherche d'officines secondaires, dont les types sont très mal connus en Égypte.

Collet R., Nenna M.-D., 2009, «L'oasis du verre », Les Métiers de l'archéologie, vol. 11, film 22 mn, Alexandrie (en français et en anglais).

Nenna M.-D., Sous presse, « Primary Glass workshops in GraecoRoman Egypt: Preliminary Report on the Excavations of the Site of Beni Salama, Wadi Natrun (2003, 2005-2009) ", in Freestone I, BAILEY J., JACKSON C.M. (ÉD.), Glass in the Roman Empire, in honour of Jennifer Price, Oxford, Oxbow books.

Nenna M.-D., 2007, « Production et commerce du verre à l'époque impériale: nouvelles découvertes et problématiques », FACTA. A Journal of Roman Material Culture, 1, p. 159-181.

NENNA M.-D., 2008, « Nouveaux acquis sur la production et le commerce du verre antique entre Orient et Occident », in Amrein H., Deschler-Erb E., Deschler-Erb S. (Éd.), Congrès International Crafts 2007: Artisanat et Société dans les provinces romaines (Zurich, 2007), Zeitschrift für Schweizerische Archäologie und Kunstgeschichte, 65.1-2, p. 61-65.

Nenna M.-D., Picon M., ViChy M., 200o, « Ateliers primaires et secondaires en Égypte à l'époque gréco-romaine », in NENNA M.-D. (ÉD.), La Route du verre: Ateliers primaires et secondaires du second millénaire av. J.-C. au Moyen Âge, Lyon, Travaux de la Maison de l'Orient, ${ }^{\circ} 33$, p. 97-112.

Nenna M.-D., Picon M., Thirion-Merle V., Vichy M., 2005, "Ateliers primaires du Wadi Natrun: nouvelles découvertes ", Annales du $16^{e}$ congrès de l'Association Internationale pour l'Histoire $d u$ Verre (Londres, 2003), Nottingham, p. 59-63.

\section{La fouille des ateliers du secteur 2}

$$
\begin{aligned}
& \text { Aurore Louis } \\
& \text { Inrap }
\end{aligned}
$$

Ma participation en tant qu'archéologue de l'Inrap à cette mission scientifique dans le Wadi Natrun pour la fouille d'un atelier de verre du Haut Empire repose sur deux points : d'un côté, mes connaissances sur la nature du site fouillé (techniques de fabrication de la matière brute et méthodes de façonnage, typologies, modes et voies de diffusion, habitudes de consommation) et, de l'autre, mes compétences en archéométrie (compositions chimiques du matériau). Ces acquis permettent de mieux appréhender les produits employés, les processus de transformation des composants et la constitution des unités de production.

La fouille des ateliers de verriers s'est avérée complexe, parfois difficile, tant techniquement que scientifiquement. Les conditions climatiques liées au milieu désertique (fortes chaleurs, tempêtes de sable) ont régulièrement ralenti les travaux. Les variations de température et d'humidité ont également eu un impact sur la conservation des structures - notamment les murs en briques crues qui disparaissent très rapidementce qui oblige à fouiller de façon très rapide.

L'enjeu était d'autant plus grand que des ateliers primaires romains étaient mis au jour en Égypte pour la première fois. Aucune comparaison, aucun parallèle avec d'autres structures similaires ne peut être fait. Le travail de documentation sur les fours primaires plus tardifs découverts en Israël et sur les ateliers secondaires d'Europe a permis de se faire une idée globale de l'organisation de ces structures artisanales. Cependant, les ateliers égyptiens se sont révélés relativement différents et la fouille n'a pu être conduite selon un schéma connu et préétabli. Les orientations de fouille ont dû être décidées au fur et à mesure de la découverte des structures. Ainsi, le four du secteur 2 a été appréhendé en cinq temps [Fig.4].

Le travail s'est appuyé sur les données connues par archéo-magnétisme. En 2005, la structure en agrafe (ayant répondu positivement aux tests) ainsi que les structures alentours ont été dégagées et relevées en plan. Ce travail préliminaire a permis d'élaborer un premier plan d'ensemble du secteur, de distinguer plus nettement les contours du four et de discerner le mode d'organisation des structures annexes. Des tests ont ensuite été réalisés au cœur du four, révélant le bassin de fusion, ses parois vitrifiées et son sol marqué des traces laissées par les outils lors de l'extraction de la dalle de verre. Ces sondages ont également permis de déterminer les différentes séquences d'abandon de la structure et surtout de noter la récupération complète du mur occidental du bassin de fusion. Cette découverte s'est avérée importante : elle a permis de comprendre qu'à la fin de la fusion l'un des longs côtés du four devait être enlevé pour faciliter l'accès à la dalle. La suite de la fouille s'est orientée vers les techniques de construction de ce bassin régulièrement démantelé et reconstruit et, surtout, vers la recherche de structures sṕécifiques à cette construction et placées sur la face orientale du bassin.

En 2006, le dégagement en plan du secteur a été poursuivi ; il a révélé la continuation de l'atelier vers le nord et vers l'ouest. Il a permis de mieux cerner le plan du bassin de fusion et de révéler la présence d'une extension riche en cendres correspondant à la chambre de chauffe. Au sud, un sondage réalisé au droit du mur du bassin a montré que ce dernier était ceinturé par un mur de briques crues, régulièrement rehaussé (au gré des différentes cuissons) et que cette partie du four était enterrée.

Au cours de l'année 2007, le dégagement du bassin de fusion et de la chambre de chauffe a été approfondi, tandis que l'aire de fouille a été élargie sur les flancs est et ouest du four. Ces agrandissements ont permis de découvrir les structures bordant le mur ouest du bassin de fusion et de mettre au jour un second bassin de fusion accolé à la face est du premier. Les relations stratigraphiques entre ces deux bassins ont 


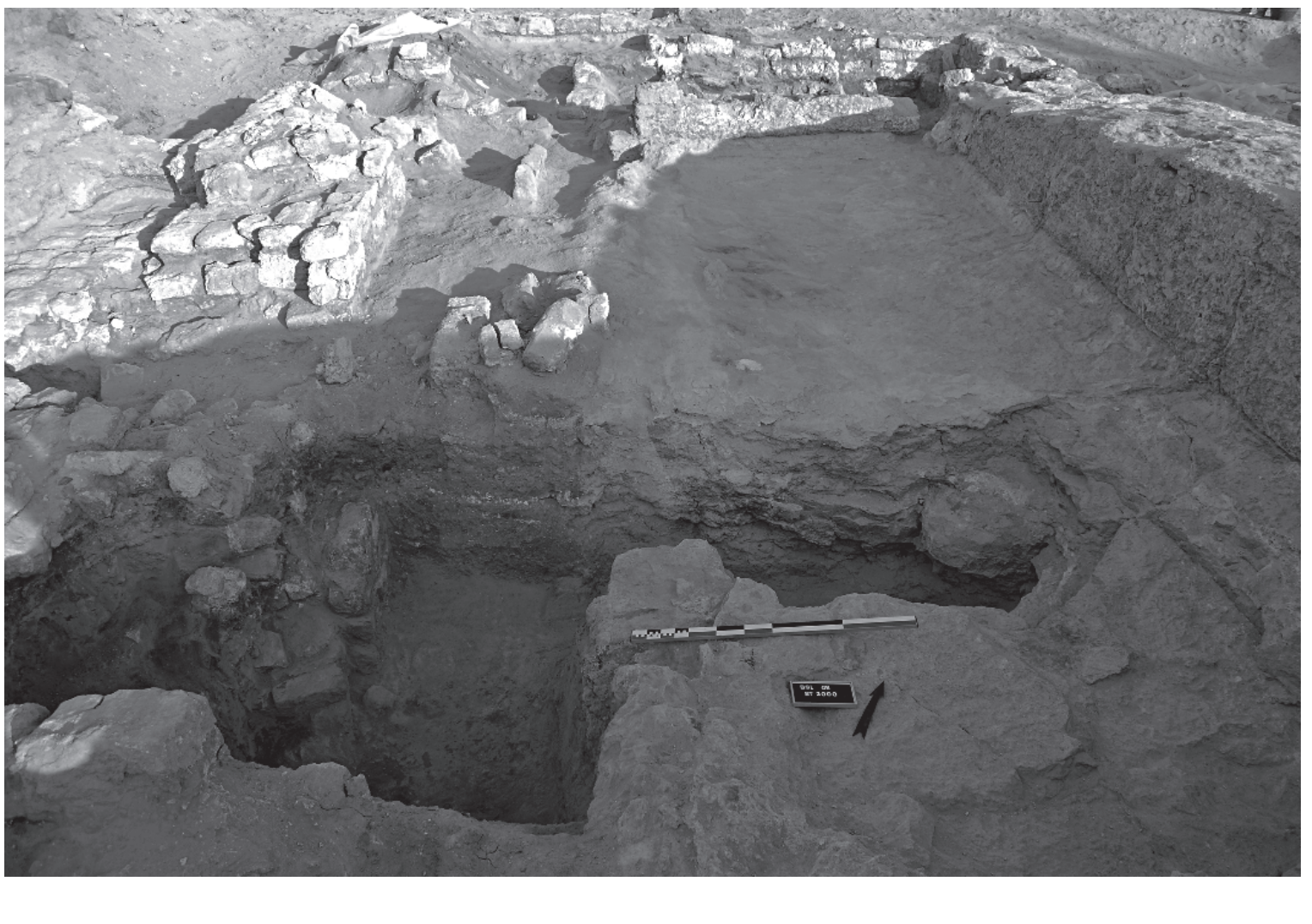

[Fig.4] Vue du sondage effectué dans et à proximité du four occidental

du secteur 2, qui a révélé les trois

phases d'activité verrière et les phases de construction et de démantèlement du mur ouest du bassin. 
confirmé ensuite qu'ils ne fonctionnaient pas en même temps et que le four oriental semble antérieur au four principal. La partie occidentale de l'aire de fouille a également révélé des structures inattendues. Il s'agit de plusieurs massifs alignés en bordure du mur récupéré ; ces maçonneries ont été interprétées comme des rampes facilitant l'accès à la voûte du bassin pour le déversement des matières brutes et le contrôle de la cuisson. Cette zone, très soignée, est dallée de briques, témoignant ainsi d'une aire de travail «propre» à fort passage. La densité des vestiges découverts au fur et à mesure de la fouille a contraint, en plus des objectifs définis, à travailler sur les nouvelles découvertes, imposant un rythme de travail plus soutenu.

Les orientations de l'année 2008 visaient à l'aboutissement du programme quinquennal en répondant au maximum de questions soulevées par ces structures. Ainsi, les trois semaines dédiées à l'achèvement de la fouille du secteur 2 ont visé à la compréhension des différents états de construction des structures, des modes d'utilisation de la chambre de chauffe et de l'évolution chronologique de la zone. Pour ce faire, une coupe transversale de la partie sud du secteur a été réalisée suivant un axe est-ouest. Elle a offert la totalité de la séquence stratigraphique de cette zone et permis d'appréhender les relations chronologiques entre le bassin de fusion, les rampes d'accès, l'espace dallé et, par extension, le bassin oriental. Une seconde coupe a été implantée dans la chambre de chauffe afin de comprendre le mode de construction de la bordure et du mur est de l'atelier, et de compléter les connaissances sur la chambre de chauffe, ainsi que sa séquence chronologique.

La campagne 2008 a marqué l'arrêt de la fouille du secteur 2. La phase d'étude des données de terrain a commencé en 2009 en vue d'une publication des résultats en 2012. Pour la post-fouille, il s'est avéré indispensable de terminer la base de données (qui combine unités stratigraphiques, faits, mobilier, documentation photographique et graphique). Cette base a permis d'élaborer plus facilement le diagramme stratigraphique du secteur 2. Cette tâche fut la plus longue à mettre en œuvre. L'arbre stratigraphique offre une organisation visuelle du secteur permettant par la suite de confondre unités et structures pour retracer le développement de l'atelier tout au long de sa période de fonctionnement. Ces données sont ensuite couplées aux facteurs graphiques. Dans un premier temps, les relevés en coupe ont été regroupés suivant leur proximité de localisation et leur position en altitude, afin de restituer la succession des structures et des unités stratigraphiques ; les résultats ont été ensuite comparés au diagramme pour validation.

Ainsi, cinq horizons chronologiques ont été définis, qui correspondent aux utilisations successives de l'atelier et se divisent en plusieurs phases (construction des structures, occupation ou utilisation, puis destruction ou abandon). Le report des relevés en coupe sur le plan d'ensemble permet ensuite de transposer les horizons chronologiques définis par la stratigraphie à l'ensemble des structures composant le secteur 2 . Il offre alors une vision générale du développement de l'atelier. La datation relative des unités stratigraphiques est ensuite confrontée à la datation absolue issue du mobilier. Celui-ci se résume à des fragments de céramiques communes et d'amphores qui ne peuvent être datées précisément dans la fourchette de trois siècles d'utilisation de ce secteur, en raison de l'absence de chronotypologies bien établies. Des prélèvements de cendres et de terre cendreuse ont été effectués afin de préciser la nature des combustibles employés et obtenir des datations au carbone 14 (laboratoire de l'Ifao).

\section{De Reqem à Pétra : investigations au cœur de la Cité rose}

\author{
François Renel \\ Inrap, UMR 7041 "Archéologies et Sciences de l'Antiquité», Aphor \\ Christian Augé \\ CNRS, IFPO \\ Mehdi Belarbi \\ Inrap \\ Xavier Peixoto \\ Inrap
}

$\mathrm{M}$

entionnée brièvement dans les textes aux $\mathrm{IV}^{\mathrm{e}}-\mathrm{III}^{\mathrm{e}}$ siècles avant notre ère, Reqem ou Pétra, cité caravanière et capitale d'un royaume unifié, connaît une longue histoire marquée par la royauté nabatéenne puis par l'intégration à l'Empire romain en 106 de notre ère, mettant fin à son statut d'État-client. Le tremblement de terre de 363 marque un tournant décisif dans le développement de la cité qui sombrera par la suite progressivement dans l'oubli au moment de l'avènement de l'islam. Redécouverte en 1812 par le Suisse Johann Burckhardt, la ville antique de Pétra a depuis fait l'objet de recherches scientifiques dès le milieu du XIX ${ }^{\mathrm{e}}$ siècle, poursuivies et accélérées grâce au développement de l'archéologie moderne à partir de la seconde moitié $\mathrm{du} \mathrm{Xx}^{\mathrm{e}}$ siècle. Paradoxalement, au-delà de ces grandes lignes, son histoire reste très mal connue : les sources épigraphiques demeurent quasi-absentes et les quelques textes des auteurs anciens ne retranscrivent pas, ou de façon tronquée, ce que l'archéologie met au jour depuis une vingtaine d'années.

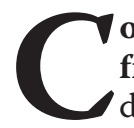
omplexité contextuelle des fouilles françaises à Pétra. Les fouilles françaises de Pétra, partie intégrante du programme «De Pétra à wadi Ramm, le sud jordanien nabatéen et arabe» (dirigé par Christian Augé), visent à étudier l'évolution d'une partie du centre de la ville antique dominée par l'implantation du sanctuaire dit «Qasr al-Bint Firaoun». Celui-ci, dédié à Dushara, dieu principal de la cité, est cerné par tout un ensemble d'édifices relatifs au culte et à sa gestion [Fig.1]. Les dégagements menés depuis 1999 montrent que, si la structuration actuelle de l'espace
1 Diodore, II, 48 et XIX 94-100. Les Nabater
sont cités comme des Bédouins nomades. 